\title{
INTERFACE EFFECTIVE PINNING AND DYNAMICAL COUPLING OF SPIN WAVES IN BILAYER FERROMAGNETIC FILMS WITH CANTED MAGNETIZATIONS
}

\author{
H. Puszkarski* and M.G. Cottam \\ Centre for Chemical Physics and Department of Physics, University of Western \\ Ontario, London, Ontario, Canada N6A 3K7 \\ (Received May 31, 1990)
}

\begin{abstract}
We present a theory for the spin-wave excitations in bilayer magnetic systems with exchange coupling across the interface between two ferromagnetic films having the same crystallographic structure. The Hamiltonian includes exchange and anisotropy terms within each magnetic film of the composite system, as well as at the interface and at the free surfaces. We examine, in particular, the effect of interface canting between the magnetization directions for each film, and we derive effective pinning parameters and dynamic coupling parameters for the interface as functions of the canting angle. We show that, in the special case when the magnetization directions are antiparallel in the bilayer system, the two films may be considered as effectively decoupled as regards the spin-wave dynamics. We discuss the relevance of this theory to recent experimental studies of bilayer ferromagnetic systems that exhibit antiferromagnetic interface exchange.
\end{abstract}

PACS numbers: 75.70.Cn, 75.30.Ds

\section{Introduction}

For a long time now there has been an interest in the properties of multilayer magnetic films (e.g. see [1] for a review of the earlier work). However, in the last few years there has been an upsurge of activity concerning these structures. This is partly due to recent developments in materials science enabling the preparation of

*Permanent address: Surface Physics Division, Physics Institute, A. Mickiewicz University, Matejki 48/49, 60-769 Poznań, Poland. 
high-quality multilayered magnetic films, including superlattices, and partly due to the development of sensitive experimental techniques to characterize the multilayers. In particular, the spin-wave properties of magnetic multilayers have attracted a considerable amount of theoretical interest for various different geometries and magnetic models (e.g., [2-8]). On the experimental side the studies of spin waves in multilayers have included using ferromagnetic resonance [9-11], spin-wave resonance [12,13], light scattering [14-17], and spin-polarized low-energy electron diffraction $[18,19]$.

In this paper we present a theoretical study of a simple multilayer structure composed of just two ferromagnetic layers (of different magnetic materials having the same crystallographic structure) coupled by exchange interactions at their interface. We are particularly concerned with investigating some novel features of the spin-wave excitations that may occur when the directions of static magnetization in each film are canted with respect to one another, and such systems have already been fabricated (see later). The energies and the amplitudes of the spin waves in this bilayer system are calculated under different conditions of interface coupling. We include the effects of exchange and anisotropy within each constituent layer as well as modified exchange interactions and modified anisotropy parameters at the interface and at the free surfaces.

In Section 2 we present the theoretical model for the bilayer ferromagnetic system. This may be regarded as an extension of a previous paper [8] concerned specifically with bulk conditions necessary for the observation of standing spin-wave resonance in bilayer ferromagnetic films. The formalism is extended here by including anisotropy effects (within each film and at the surfaces and interface) and propagation of the spin waves parallel to the plane of the films. In fact, the parallel propagation leads to a richer spectrum for the spin-wave excitations of the coupled system, and this would be relevant for experimental studies. The derivation of the spin-wave energies and amplitudes are given first in Section 3 using a microscopic theory valid for all wavectors. Then in Section 4 we present a continuum theory appropriate in the case of small-wavevector (long-wavelength) excitations only, and the connexion between the two methods is established. The latter approach is particularly useful in gaining a physical interpretation of the boundary conditions (at the surfaces and interface). In Section 5 we analyze the spin-wave results in terms of effective interface coupling and pinning parameters, and special cases of the canting angle between magnetization directions are discussed.

\section{The model}

We consider a bilayer system composed of two ferromagnetic materials (labeled $A$ and $B$ ) in the form of parallel-sided thin films, and Cartesian axes are chosen as indicated in Fig. 1(a). Each layer is assumed to have a homogeneous structure and to extend indefinitely in the directions parallel to the surfaces (so that there is translational symmetry in the $x y$ plane). In general, $A$ and $B$ may have different magnetic properties (in terms of the magnitudes and directions of magnetization, the spin quantum numbers, and the exchange and anisotropy 
(b)
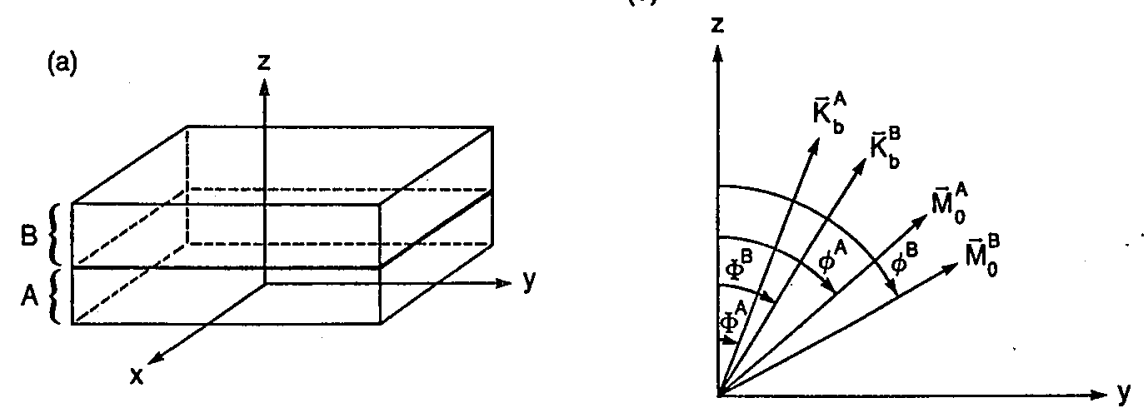

Fig. 1. The assumed geometry: (a) the bilayer system, consisting of two ferromagnetically ordered films $A$ and $B$, and the choice of $x, y, z$ axes; (b) the orientation in the $y z$ plane of the film magnetizations and bulk anisotropies.

parameters). However, for simplicity we shall assume that their crystallographic structures are identical. The bilayer sample forms one coupled magnetic system because of the exchange interactions assumed to exist across the interface between the films $A$ and $B$.

With the above assumptions we may consider the bilayer system as made up of a number of lattice planes perpendicular to the $z$ axis. Each plane will have identical crystallographic symmetry and the separation of the planes will be a constant denoted by $a_{0}$. Any magnetic ion will be specified by the set of indices $\ell, j$, where $\ell$ is an integer labeling the plane containing the ion and $j$ is a two-dimensional lattice vector in the $x y$ plane. As shown in Fig. 2 the integer $\ell$ ranges in value from 0 to $L-1$, corresponding to a total thickness of $(L-1) a_{0}$ for the bilayer system, as follows:

$$
\begin{array}{ll}
\ell=0 & : \text { Surface plane of } A \\
\ell=1,2, \ldots, N-2 & : \text { Internal planes of } A \\
\ell=N-1 & : \text { Interface plane of } A \\
\ell=N & : \text { Interface plane of } B \\
\ell=N+1, N+2, \ldots, L-2 & : \text { Internal planes of } B \\
\ell=L-1 & : \text { Surface plane of } B
\end{array}
$$

We first consider the static magnetizations within the bilayer system by employing a low-temperature semiclassical approximation for the spins. We assume the spins in films $A$ and $B$ to be acted on by bulk anisotropy fields $K_{b}^{A}$ and $\boldsymbol{K}_{b}^{B}$, respectively, which are independent of position within each film. In addition there are exchange interactions which we discuss later in terms of an isotropic Ireisenberg model. The anisotropy fields may be tilted away from the $z$ direction through angles $\Phi^{A}$ and $\Phi^{B}$, but for simplicity we assume them at this stage to lie in the same vertical plane (see Fig. 1(b)), taken as the $y z$ plane. The effective bulk anisotropy field $H_{b}^{n}(n=A, B)$ is obtained by taking account of the 


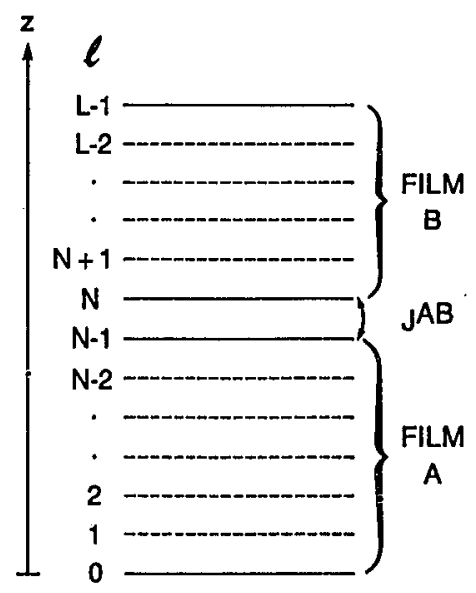

Fig. 2. Schematic illustration of the bilayer system, showing film $A$ with layer index $\ell$ ranging from 0 to $N-1$ and film $B$ with $\ell$ ranging from $N$ to $L-1$; the nearest neighbor exchange coupling across the interface between layers $N-1$ and $N$ is denoted by $J^{A B}$.

shape-dependent demagnetization in each film:

$$
\boldsymbol{H}_{b}^{n}=\boldsymbol{K}_{b}^{n}-i_{z} 4 \pi M_{0}^{n} \cos \phi^{n} \quad(n=A, B),
$$

where $i_{z}$ is a unit vector in the $z$ direction, $M_{0}^{n}$ is the magnitude of the static magnetization in film $n$ (taken to be homogeneous because of the previous assumption concerning $\boldsymbol{K}_{b}^{n}$ ), and $\phi^{n}$ specifies the magnetization direction (Fig. 1(b)). In principle the angles $\phi^{A}$ and $\phi^{B}$ are determined by applying a minimization procedure to the total magnetic energy of the coupled bilayer system. However, in a first approximation we may assume that they can be found by minimizing the energies for each of the films separalely. The resulting directions of $M_{0}^{n}$ will be parallel to the respective effective fields defined in (1), and the tilts $\phi^{A}$ and $\phi^{B}$ of the magnetizations can be found from the standard equations [1]:

$$
\frac{K_{b}^{n}}{4 \pi M_{0}^{n}}=\frac{\sin \left(2 \phi^{n}\right)}{2 \sin \left(\phi^{n}-\Phi^{n}\right)} \quad(n=A, B)
$$

The canting angle $\delta$ between the static magnetization directions is therefore $\left|\phi^{A}-\phi^{B}\right|$.

We carry out our calculations of the spin-wave properties within the framework of the Heisenberg localized spin model. With the inclusion of exchange and Zeeman terms the Hamiltonian may be expressed in the form:

$$
\mathcal{H}=-\sum_{\ell, j, \ell^{\prime}, j^{\prime}} J_{\ell \ell^{\prime}}, S_{\ell, j} \cdot S_{\ell^{\prime}, j^{\prime}}-g \mu_{B} \sum_{\ell, j} H_{\ell}^{\mathrm{eff}} \cdot S_{\ell, j^{\prime}}
$$

where the summations in the first term are taken over pairs of nearest neighbor spins only. For a spin layer $\ell$ the nearest neighbors will all be in the same or 
adjacent layers, so that $\ell^{\prime}$ is restricted to the values $\ell-1, \ell$, and $\ell+1$. We make the following assumptions for the nearest neighbor exchange terms: $J_{\ell \ell^{\prime}}=J^{A}$ if both spins are in film $A, J_{\ell \ell^{\prime}}=J^{B}$ if both spins in film $B$, and $J_{\ell \ell^{\prime}}=J^{A B}$ for spins coupled across the interface between $A$ and $B$. The quantity $\boldsymbol{H}_{\ell}^{\text {eff }}$ in the second term of (3) is an effective anisotropy field acting on spins in layer $\ell$. Its magnitude and direction will depend on the anisotropy and on the magnetization in each film, and we take it to be specified as follows:

$$
\boldsymbol{H}_{\ell}^{\text {eff }}= \begin{cases}\boldsymbol{H}_{b}^{A}+\boldsymbol{K}_{s}^{A} & \text { for } \ell=0 \text { (surface plane of } A), \\ \boldsymbol{H}_{b}^{A} & \text { for } 0<\ell<N-1, \\ \boldsymbol{H}_{b}^{A}+K_{i}^{A} & \text { for } \ell=N-1 \text { (interface plane of } A), \\ \boldsymbol{H}_{b}^{B}+\boldsymbol{K}_{i}^{B} & \text { for } \ell=N \text { (interface plane of } B), \\ \boldsymbol{H}_{b}^{B} & \text { for } N<\ell<L-1, \\ \boldsymbol{H}_{b}^{B}+K_{s}^{B} & \text { for } \ell=L-1 \text { (surface plane of } B \text { ). }\end{cases}
$$

Here $\boldsymbol{H}_{b}^{A}$ and $\boldsymbol{H}_{b}^{B}$ are the bulk fields defined in (1), while $\boldsymbol{K}_{s}^{A}$ and $\boldsymbol{K}_{s}^{B}$ are surface anisotropy fields assumed to exist at the surface planes of $A$ and $B$, respectively, and correspondingly $\boldsymbol{K}_{i}^{A}$ and $\boldsymbol{K}_{i}^{B}$ are interface anisotropy fields. The introduction of surface and interface anisotropy terms will be shown to be important for the spin-wave dynamics (by modifying the boundary conditions), even though we neglect these quantities in establishing the bulk static properties. By writing the Hamiltonian as in (3) we are taking the magnetic dipcle-dipole terms to be negligible for the spin-wave properties (except in giving a static demagnetizing field). This is a good approximation provided the wavevector of the spin waves is not too small (e.g., see [17]).

\section{Microscopic theory of spin waves}

The spin-wave excitations in the bilayer system may be calculated using the Hamiltonian (3) by a direct generalization of the approach detailed in Ref. [20] for a single magnetic film. Essentially the method consists of transforming the spin Hamiltonian to a representation in terms of boson operators (appropriate to the low temperature regime). A further operator transformation can then be applied to 'diagonalize' the Hamiltonian to a quasiparticle form. This yields both the spin-wave energies and the spatial dependence of the eigenfunctions that give the spin-wave amplitude (in the circular spin-precession approximation) within the finite magnetic system. In the present application to the bilayer system we need to take account of the two coupled films with different magnetic properties, the canting of the magnetizations, and the interface region. Consequently, the detailed calculations are more complicated, but nevertheless they proceed in an analogous fashion to [20] and we quote only the main results here.

We denote the wavevector component of the spin waves parallel to the surfaces by $k_{\|}=\left(k_{x}, k_{y}\right)$. The bulk spin waves (including standing bulk spin waves) will additionally be characterized by a third wavevector component $k_{z}$ in the $z$ direction. The characteristic equation determining the values of $k_{z}$, which will generally be different in the two films, is obtained from the diagonalization procedure 
and is described later. Utilizing the property of translation invariance parallel to the surface we may express the spin-wave eigenfunctions in the form:

$$
\Psi_{\ell, j}\left(k_{\|}, k_{z}\right)=U_{\ell}\left(k_{\|}, k_{z}\right) \exp \left[\mathrm{i}\left(k_{\|} \cdot j\right)\right] .
$$

Any localized surface or interface spin waves will be characterized by $k_{\|}$and one or more decay constants, and by analogy with earlier work on surface modes [2, 3 ] the real $k_{z}$ in (5) then becomes replaced by a complex quantity describing the decay (see later). It is also convenient at this stage to define some factors that depend on the assumed crystallographic structure:

$$
\gamma_{\|}\left(k_{\|}\right)=\frac{1}{z_{\|}} \sum_{\delta_{\|}} \operatorname{expi}\left(k_{\|} \cdot \delta_{\|}\right)
$$

and

$$
\gamma_{\perp}\left(k_{\|}\right)=\frac{1}{z_{\perp}} \sum_{\delta_{\perp}} \exp \mathrm{i}\left(k_{\|} \cdot \delta_{\perp}\right)
$$

where $\delta_{\|}$denotes any vector connecting a site in layer $\ell$ with its nearest neighbors in the same layer, and $\delta_{\perp}$ denotes the projection in the $x y$ plane of any vector connecting a site in layer $\ell$ with its nearest neighbors in layer $\ell+1$. Also $z \|$ and $z_{\perp}$ denote the number of nearest neighbors in each case. We note that $\gamma_{\|}\left(k_{\|}\right)$is always real for two-dimensional layers having inversion symmetry, but $\gamma_{\perp}\left(k_{\|}\right)$may be complex for some lattice structures and surface orientations. We introduce a phase term $\zeta\left(k_{\|}\right)$by writing:

$$
\gamma_{\perp}\left(\boldsymbol{k}_{\|}\right)=\left|\gamma_{\perp}\left(\boldsymbol{k}_{\|}\right)\right| \exp \left[\mathbf{i} \zeta\left(\boldsymbol{k}_{\|}\right)\right] .
$$

In the special case of $k_{\|}=0$, we have $\gamma_{\perp}\left(k_{\|}\right)$real and so $\zeta\left(k_{\|}\right)=0$.

If the spin-wave amplitude $U_{\ell}\left(k_{\|}, k_{z}\right)$ in layer $\ell$ is transformed by setting [20]:

$$
U_{\ell}\left(k_{\|}, k_{z}\right)=u_{\ell}\left(k_{z}\right) \exp \left[\mathrm{i} \zeta\left(k_{\|}\right) \ell\right],
$$

we find from the diagonalization procedure that the terms $u_{\ell}\left(k_{z}\right)$ satisfy the following set of homogeneous finite-difference equations:

$$
\begin{gathered}
\left(x_{A}-d_{A}\right) u_{0}-u_{1}=0 \quad(\ell=0) \\
x_{A} u_{\ell}-u_{\ell-1}-u_{\ell+1}=0 \quad(0<\ell<N-1) \\
\left(x_{A}-b\right) u_{N-1}-u_{N-2}-\rho u_{N}=0 \quad(\ell=N-1) \\
\left(x_{B}-c\right) u_{N}-\rho^{\prime} u_{N-1}-u_{N+1}=0 \quad(\ell=N) \\
x_{B} u_{\ell}-u_{\ell-1}-u_{\ell+1}=0 \quad(N<\ell<L-1) \\
\left(x_{B}-d_{B}\right) u_{L-1}-u_{L-2}=0 \quad(\ell=L-1) .
\end{gathered}
$$

Here we have introduced the following notation (with $n=A, B$ ):

$$
x_{n}=\frac{1}{z_{\perp}\left|\gamma_{\perp}\left(k_{\|}\right)\right|}\left[2 z_{\perp}+z_{\|}\left[1-\gamma_{\|}\left(k_{\|}\right)\right]+\frac{1}{2 S^{n} J^{n}}\left(g \mu_{B} H_{b}^{n} \cdot m^{n}-E\right)\right],
$$




$$
d_{n}=\frac{1}{z_{\perp}\left|\gamma_{\perp}\left(k_{\|}\right)\right|}\left[z_{\perp}-\frac{g \mu_{B}}{2 S^{n} J^{n}}\left(K_{s}^{n} \cdot m^{n}\right)\right]
$$

and

$$
\begin{gathered}
b=\frac{1}{z_{\perp}\left|\gamma_{\perp}\left(k_{\|}\right)\right|}\left[z_{\perp}-\frac{g \mu_{B}}{2 S^{A} J^{A}}\left(K_{i}^{A} \cdot m^{A}\right)-\frac{S^{B} J^{A B}}{S^{A} J^{A}}\left(z_{\perp} m^{A} \cdot m^{B}\right)\right] \\
c=\frac{1}{z_{\perp}\left|\gamma_{\perp}\left(k_{\|}\right)\right|}\left[z_{\perp}-\frac{g \mu_{B}}{2 S^{B} J^{B}}\left(K_{i}^{B} \cdot m^{B}\right)-\frac{S^{A} J^{A B}}{S^{B} J^{B}}\left(z_{\perp} m^{A} \cdot m^{B}\right)\right] \\
\rho=\frac{1}{2}\left(\frac{S^{B}}{S^{A}}\right)^{1 / 2} \frac{J^{A B}}{J^{A}}\left(1+m^{A} \cdot m^{B}\right) \\
\rho^{\prime}=\frac{1}{2}\left(\frac{S^{A}}{S^{B}}\right)^{1 / 2} \frac{J^{A B}}{J^{B}}\left(1+m^{A} \cdot m^{B}\right)
\end{gathered}
$$

In the above expressions $S^{A}$ and $S^{B}$ denote the spin quantum numbers corresponding to films $A$ and $B$, respectively, $m^{A}$ and $m^{B}$ are unit vectors in the directions of the static magnetizations in films $A$ and $B$, and $E$ is the energy of a spin wave. The quantities $d_{A}$ and $d_{B}$ play the role of surface pinning parameters, while $b$ and $c$ are analogous interface pinning terms. We note from (13)-(16) that the latter quantities and the interface coupling parameters $\rho$ and $\rho^{\prime}$ depend on the canting angle $\delta$ through the factor $m^{A} \cdot m^{B} \equiv \cos \delta$. This dependence will turn out to have important physical consequences, as we discuss later.

We emphasize at this stage that the results given in (10)-(16) for the coupled equations are, in fact, of wider validity than for the geometry of Fig. 1(b), where the bulk anisotropy fields and static magnetizations are all in the $y z$ plane. In particular, it may be shown that the same formulae also apply when the bulk anisotropy fields and the magnetizations are in the $x y$ plane (parallel to the surfaces). However, in that case the magnetizations in each film are aligned along the respective bulk anisotropy field, and so there is no need to solve an equation analogous to (2) to establish the equilibrium conditions. The canting angle $\delta$ becomes the same as the angle between the directions of the bulk anisotropy fields in $A$ and $B$.

Equations (10)-(16) will now be used to discuss the solutions for the spin-wave modes of the bilayer system. The set of Eqs. (10) has already been solved exactly by one of us [21] by applying the so-called Recurrential Interface Rescaling method especially developed for that purpose in a multilayer system. Subsequently, the results obtained [21] were re-expressed in equivalent but simpler forms [22], and they will be used in our following analysis. The method is a generalization of the long-established formalism [23] for solving the corresponding set of finite-difference equations in the case of a single ferromagnetic film.

First we define the quantities $k_{A}$ and $k_{B}$ related to each film by

$$
x_{A}=2 \cos \left(k_{A} a_{0}\right) ; \quad x_{b}=2 \cos \left(k_{B} a_{0}\right),
$$


where $a_{0}$ is the interlayer distance. For the case of bulk spin waves (standing modes) in each film, $k_{A}$ and $k_{B}$ are real and have the simple interpretation of being the $z$-component $k_{z}$ of the wavevector in the respective films. The corresponding spin-wave energy $E$ can be expressed in terms of either of these quantities using (11):

$$
\begin{array}{r}
E\left(k_{\|}, k_{A}\right)=g \mu_{B}\left(\boldsymbol{H}_{b}^{A} \cdot m^{A}\right)+2 S^{A} J^{A}\left[2 z_{\perp}+z_{\|}\left(1-\gamma_{\|}\left(k_{\|}\right)\right)\right] \\
-4 S^{A} J^{A} z_{\perp}\left|\gamma_{\perp}\left(k_{\|}\right)\right| \cos \left(k_{A} a_{0}\right)
\end{array}
$$

or

$$
\begin{aligned}
E\left(k_{\|}, k_{B}\right)=g \mu_{B}\left(H_{b}^{B} \cdot m^{B}\right)+ & 2 S^{B} J^{B}\left[2 z_{\perp}+z_{\|}\left(1-\gamma_{\|}\left(k_{\|}\right)\right)\right] \\
- & 4 S^{B} J^{B} z_{\perp}\left|\gamma_{\perp}\left(k_{\|}\right)\right| \cos \left(k_{B} a_{0}\right) .
\end{aligned}
$$

By equating the right-hand sides of (18) and (19) we arrive at a consistency condition to be satisfied by $k_{A}$ and $k_{B}$ :

$$
\begin{aligned}
& 2\left(S^{A} J^{A}-S^{B} J^{B}\right)\left\{2 z_{\perp}+z_{\|}\left[1-\gamma_{\|}\left(k_{\|}\right)\right]\right\}+g \mu_{B}\left(\boldsymbol{H}_{b}^{A} \cdot \boldsymbol{m}^{A}-\boldsymbol{H}_{b}^{B} \cdot \boldsymbol{m}^{B}\right) \\
&=4 z_{\perp}\left|\gamma_{\perp}\left(k_{\|}\right)\right|\left[S^{A} J^{A} \cos \left(k_{A} a_{0}\right)-S^{B} J^{B} \cos \left(k_{B} a_{0}\right)\right] .
\end{aligned}
$$

This relation can be used to eliminate either of $k_{A}$ or $k_{B}$ from subsequent expressions, if required.

In addition to (20) the wavevector components have to satisfy a characteristic equation that specifically derives from the suface and interface equations in (10). It can be written in the form:

$$
\begin{array}{r}
\sin \left[(L-N+1) k_{B} a_{0}\right]+C\left(k_{A}\right) d_{B} \sin \left[(L-N-1) k_{B} a_{0}\right] \\
-\left[C\left(k_{A}\right)+d_{B}\right] \sin \left[(L-N) k_{B} a_{0}\right]=0,
\end{array}
$$

where $C\left(k_{A}\right)$ is a rescaled [21] interface pinning parameter given by:

$$
\begin{array}{r}
C\left(k_{A}\right)=c+\rho \rho^{\prime}\left\{\sin \left(N k_{A} a_{0}\right)-d_{A} \sin \left[(N-1) k_{A} a_{0}\right]\right\}\left\{\sin \left[(N+1) k_{A} a_{0}\right]\right. \\
\left.-\left(d_{A}+b\right) \sin \left(N k_{A} a_{0}\right)+d_{A} b \sin \left[(N-1) k_{A} a_{0}\right]\right\}^{-1} .
\end{array}
$$

We have expressed (21) in the analogous form to the result for a single film [23], but with the important difference that in the present case we have $C\left(k_{A}\right)$ as a modified interface pinning parameter instead of just a surface pinning parameter for the case of a single film. We note from (20)-(22) the discrete set of allowed values of $k_{A}$ and $k_{B}$ is determined by all the pinning and coupling parameters and by both of the film thicknesses of the bilayer system.

The unnormalized exact solutions satisfying (10) can be expressed as [22]:

$$
u_{\ell}\left(k_{A}\right)=D\left(k_{A}, k_{B}\right)\left\{\sin \left[(\ell+1) k_{A} a_{0}\right]-d_{A} \sin \left(\ell k_{A} a_{0}\right)\right\}
$$

for $\ell=0,1, \ldots, N-1$, 


$$
\begin{gathered}
u_{\ell}\left(k_{B}\right)=\sin \left[(L-\ell) k_{B} a_{0}\right]-d_{B} \sin \left[(L-\ell-1) k_{B} a_{0}\right] \\
\text { for } \ell=N, N+1, \ldots, L-1, \\
D\left(k_{A}, k_{B}\right)=\rho\left\{\sin \left[(L-N) k_{B} a_{0}\right]-d_{B} \sin \left[(L-N-1) k_{B} a_{0}\right]\right\} \\
\left.\times\left\{\sin \left[(N+1) k_{A} a_{0}\right]-\left(d_{A}+b\right) \sin \left(N k_{A} a_{0}\right)+d_{A} b \sin \left[(N-1) k_{A} a_{0}\right)\right]\right\}^{-1}, \quad(25)
\end{gathered}
$$

where $z$-components of the wavevectors have to satisfy the relationships discussed above, and $d_{A}$ and $d_{B}$ are the surface parameters defined by (12). The spin-wave eigenfunctions introduced in (5) can then be written in the form:

$$
\Psi_{\ell, j}\left(k_{\|}, k\right)=\frac{\mathcal{A}}{\sqrt{L_{x y}}} \exp \left[\mathrm{i}\left(k_{\|} \cdot j+\zeta\left(k_{\|}\right) \ell\right)\right] u_{\ell}(k),
$$

where $\mathcal{A}$ is a normalization factor, $L_{x y}$ denotes the number of sites in each layer parallel to the surfaces, and $u_{\ell}(k)$ is given by (23)-(24). The quantity $k$ denotes either $k_{A}$ or $k_{B}$ depending on whether $\ell$ lies within the film $A$ or the film $B$, respectively.

\section{Long-Wavelength continuum theory of spin waves}

We now discuss an alternative formulation for the spin waves in the bilayer system by using a continuum theory valid for long-wavelength excitations. It is useful to consider this because the preceding microscopic theory then simplifies, and it leads to a more direct physical interpretation of the results. Also the long-wavelength case is of interest to facilitate comparison with some of the experimental measurements (e.g. in magnetic resonance and light scattering).

It is convenient to present the results by forming the continuum limit of the theory in Section 3. This may be done by analogy with previous spin-wave calculations for a single ferromagnetic film (e. g. see [24]) and we again quote only the main results. We go over to the continuum description by making the replacement $u_{\ell} \rightarrow u(z)$ for the spin-wave amplitude, where $z=\ell a_{0}$. For the layers adjacent to $\ell$ we make the replacements:

$$
u_{\ell \pm 1} \rightarrow u\left(z \pm a_{0}\right) \approx u(z)+a_{0} \frac{\partial u}{\partial z} \pm \frac{1}{2} a_{0}^{2} \frac{\partial^{2} u}{\partial z^{2}}
$$

where higher order terms in the Taylor series expansion are neglected, and the derivatives are evaluated at coordinate $z$ (treated now as a continuous variable). In practice, this will be a good approximation provided $k_{A} a_{0} \ll 1$ and $k_{B} a_{0} \ll 1$ for standing bulk spin waves (where $k_{A}$ and $k_{B}$ denote $z$-component wavevectors in the films $A$ and $B$, as before). For localized spin waves, the validity requirement becomes $\xi a_{0} \ll 1$, where $\xi$ represents any characteristic attenuation factor (reciprocal decay length). The previous set of finite-difference equations represented by 
(10) now become replaced by their corresponding differential equations as follows (where we denote the overall thicknesses of the films $A$ and $B$ by $D_{A}$ and $D_{B}$, respectively, and each equation is expanded to lowest non-vanishing order in the derivatives of $u(z))$. Within each film we have:

$$
\begin{gathered}
\left(x_{A}-2\right) u-a_{0}^{2} \frac{\partial^{2} u}{\partial z^{2}}=0 \text { for } 0<z<D_{A}, \\
\left(x_{B}-2\right) u-a_{0}^{2} \frac{\partial^{2} u}{\partial z^{2}}=0 \text { for } D_{A}<z<D_{A}+D_{B} .
\end{gathered}
$$

The boundary conditions at the outer surfaces of the bilayer are:

$$
\begin{gathered}
\left(x_{A}-d_{A}-1\right) u \cdots a_{0} \frac{\partial u}{\partial z}=0 \quad \text { at } z=0, \\
\left(x_{B}-d_{B}-1\right) u+a_{0} \frac{\partial u}{\partial z}=0 \text { at } z=D_{A}+D_{B} .
\end{gathered}
$$

The boundary conditions at each side of the interface at $z=D_{A}$ are:

$$
\begin{aligned}
& \left(x_{A}-b-1\right) u^{-}+a_{0} \frac{\partial u^{-}}{\partial z}-\varrho u^{+}-\varrho a_{0} \frac{\partial u^{+}}{\partial z}=0, \\
& \left(x_{B}-c-1\right) u^{+}-a_{0} \frac{\partial u^{+}}{\partial z}-\varrho^{\prime} u^{-}+\varrho^{\prime} a_{0} \frac{\partial u^{-}}{\partial z}=0,
\end{aligned}
$$

where superscripts \pm indicate that $u$ (or its derivative) are to be evaluated at the side of the interface corresponding to $z=D_{A} \pm 0^{+}$, respectively. The parameters $x_{A}, x_{B}, d_{A}, d_{B}, b, c, \rho$ and $\rho^{\prime}$ are all as defined in Section 3. However, in the long-wavelength limit the expressions of these quantities (with the exception of $\rho$ and $\rho^{\prime}$ ) simplify because we can then make the following approximate replacements for the structure factors:

$$
\begin{gathered}
\gamma_{\|}\left(k_{\|}\right) \approx 1-\mu_{\|} a^{2} k_{\|}^{2} \quad\left(a^{2} k_{\|}^{2} \ll 1\right), \\
\left|\gamma_{\perp}\left(k_{\|}\right)\right| \approx 1-\mu_{\perp} a^{2} k_{\|}^{2} \quad\left(a^{2} k_{\|}^{2} \ll 1\right)
\end{gathered}
$$

with $a$ denoting the lattice constant for the layers in the $x y$ plane. The numerical factors $\mu_{\|}$and $\mu_{\perp}$, which are structure-dependent and of order unity or less, are quoted later for the case of a simple cubic lattice and (001) surfaces.

We note that the surface boundary conditions of (30)-(31) take the well-known Rado-Weertman (RW) form [25] with terms proportional to $u$ and $\partial u / \partial z$. The interface boundary conditions of (32)-(33) also involve $u$ and $\partial u / \partial z$, but with extra terms that correspond to the exchange coupling across the interface (through parameters $\rho$ and $\rho^{\prime}$ ). In the special case of $\rho=\rho^{\prime}=0$, eqs. (32)-(33) reduce to the usual RW form (the two films then being decoupled).

The characteristic solutions for the spin-wave modes of the bilayer system may straightforwardly be deduced within this continuum approach. The general solutions of (28)-(29) for the amplitude factor $u(z)$ within each film are:

$$
u(z)=F_{n} \exp \left(\mathrm{i} k_{n} z\right)+G_{n} \exp \left(-\mathrm{i} k_{n} z\right)
$$


where $n=A$ if $0<z<D_{A}$ and $n=B$ if $D_{A}<z<D_{A}+D_{B}$. Coefficients $F_{n}$ and $G_{n}$ are to be determined by application of the surface and interface boundary conditions, and the quantities $k_{n}$ satisfy

$$
x_{n}-2=-a_{0}^{2} k_{n}^{2} \quad(n=A, B) .
$$

For the case of standing bulk modes in each film, $k_{A}$ and $k_{B}$ are real wavevector components and (37) is the long-wavelength continuum approximation to (17). The corresponding spin-wave energy is given by either of the equivalent expressions in (18)-(19) expanded to the second order in $a^{2} k_{\|}^{2}, a_{0}^{2} k_{A}^{2}$ and $a_{0}^{2} k_{B}^{2}$. In this approximation the consistency condition (20) relating $k_{A}$ and $k_{B}$ becomes:

$$
\begin{array}{r}
2\left(S^{A} J^{A}-S^{B} J^{B}\right)\left(2 z_{\perp} \mu_{\perp}+z_{\|} \mu_{\|}\right) a^{2} k_{\|}^{2}+g \mu_{B}\left(\boldsymbol{H}_{b}^{A} \cdot \boldsymbol{m}^{A}-\boldsymbol{H}_{b}^{B} \cdot \boldsymbol{m}^{B}\right) \\
=4 z_{\perp}\left(S^{A} J^{A} a_{0}^{2} k_{A}^{2}-S^{B} J^{B} a_{0}^{2} k_{B}^{2}\right) .
\end{array}
$$

This can be satisfied for small values of the wavevectors only if the term involving the anisotropy field is not too large, and this places a limitation on the generality of the continuum approach compared with the results of Section 3.

Apart from having standing bulk spin waves in each film, there is also the possibility of localized modes occurring in one or both of the films. We can still formally write $u(z)$ as in (36), but the corresponding spin-wave energy $E$ (which is real, but no longer given by (18)-(19) would be such that one (or both) of the quantities $k_{A}$ and $k_{B}$ are complex. A set of linear equations satisfied by the coefficients $F_{A}, G_{A}, F_{B}$ and $G_{B}$ are obtained on applying the boundary conditions of (30)-(31) and (32)-(33). A solvability condition can then be written down in terms of the vanishing of a $4 \times 4$ determinant, and this is just the characteristic equation for the spin waves in the bilayer system. When $k_{A}$ and $k_{B}$ are both real, we obtain results that are just the long-wavelength limit of expressions given in Section 3, as expected. The same method can also be used to deduce the dispersion relations and amplitudes for localized spin-wave modes, and we discuss this in a later paper.

\section{Effective interface coupling and pinning}

We now employ the results of Sections 3 and 4 to discuss the effective coupling and pinning at the interface for the standing bulk spin waves. It is evident from both the microscopic and the continuum approaches that the effective spin-wave coupling across the interface is described by the quantities $\rho$ and $\rho^{\prime}$ defined in (15) and (16). We note that both $\rho$ and $\rho^{\prime}$ depend on the canting angle $\delta$ through the factor $\left(1+m^{A} \cdot m^{B}\right)$ or, equivalently, $2 \cos ^{2}(\delta / 2)$. Hence, they are at a maximum when the film magnetization directions are parallel $\left(\delta=0^{\circ}\right)$, and in the case when the film magnetizations are antiparallel $\left(\delta=180^{\circ}\right)$ the films may be considered as effectively decoupled as regards their spin-wave dynamics.

We now discuss this behavior in more detail, as well as the general dependence on the angle $\delta$, by considering the specific case where the films have a simple-cubic 
lattice structure with (001) surfaces. The structure factors defined in (6) and (7) take the form:

$$
\begin{gathered}
\gamma_{\|}\left(k_{\|}\right)=\left[\cos \left(k_{x} a\right)+\cos \left(k_{y} a\right)\right] / 2, \\
\gamma_{\perp}\left(k_{\|}\right)=1,
\end{gathered}
$$

leading to simplifications in (11)-(14). Also we have $\mu_{\|}=1 / 4$ and $\mu_{\perp}=0$ for the expansion parameters in (34)-(35). For further simplicity we concentrate on the case of $\boldsymbol{k}_{\|}=0$ for the in-plane wavevector component (as in a spin-wave resonance experiment). The quantities $x_{n}, d_{n}(n=A, B), b$, and $c$ then reduce to

$$
\begin{gathered}
x_{n}=2+\frac{1}{2 S^{n} J^{n}}\left[g \mu_{B} H_{b}^{n} \cdot m^{n}-E\right], \\
d_{n}=1-\frac{g \mu_{B}}{2 S^{n} J^{n}}\left[K_{s}^{n} \cdot m^{n}\right], \\
b=1-\frac{g \mu_{B}}{2 S^{A} J^{A}}\left[K_{i}^{A} \cdot m^{A}\right]-\frac{S^{B} J^{A B}}{S^{A} J^{A}} \cos (\delta), \\
c=1-\frac{g \mu_{B}}{2 S^{B} J^{B}}\left[K_{i}^{B} \cdot m^{B}\right]-\frac{S^{A} J^{A B}}{S^{B} J^{B}} \cos (\delta)
\end{gathered}
$$

and

$$
\begin{aligned}
& \rho=\left[\frac{S^{B}}{S^{A}}\right]^{1 / 2} \frac{J^{A B}}{J^{A}} \cos ^{2}(\delta / 2), \\
& \rho^{\prime}=\left[\frac{S^{A}}{S^{B}}\right]^{1 / 2} \frac{J^{A B}}{J^{B}} \cos ^{2}(\delta / 2) .
\end{aligned}
$$

As a numerical example of the effect of varying the canting angle $\delta$, Fig. 3 shows the dependence of the effective interface coupling parameters $\rho$ and $\rho^{\prime}$ (broken curves) taking arbitrarily the case of $S^{A}=S^{B}$ for the spins. As commented earlier, the interface spin-wave coupling vanishes for $\delta=180^{\circ}$ and has its maximum effect for $\delta=0^{\circ}$ (or $360^{\circ}$ ). We also show in Fig. 3 the corresponding variations of $b$ and $c$ (full curves) with angle $\delta$, neglecting for simplicity the interface anisotropy fields $K_{i}^{A}$ and $K_{i}^{B}$. The quantities $b$ and $c$ play the same role at the interface as do the pinning parameters $d_{A}$ and $d_{B}$ at the surfaces of the film (e. g. see (10) for the microscopic theory or (30)-(33) for the continuum theory): they may therefore be termed effective interface pinning paramelers. We see from Fig. 3 that the $\delta$-dependences of the interface coupling parameters and pinning parameters are complementary in the sense that decreases in $\rho$ and $\rho^{\prime}$ are accompanied by increases in $b$ and $c$, and vice versa.

Figure 4 illustrates the changes in the interface pinning and spin-wave coupling parameters when the interface exchange $J^{A B}$ is varied over positive and negative values. The results are plotted for $b$ and $\rho$ versus the ratio $J^{A B} / J^{A}$ in Fig. 4(a) and 4(b), respectively, for three values of the canting angle corresponding to the magnetizations being parallel, perpendicular, or antiparallel. We assume 
(a)

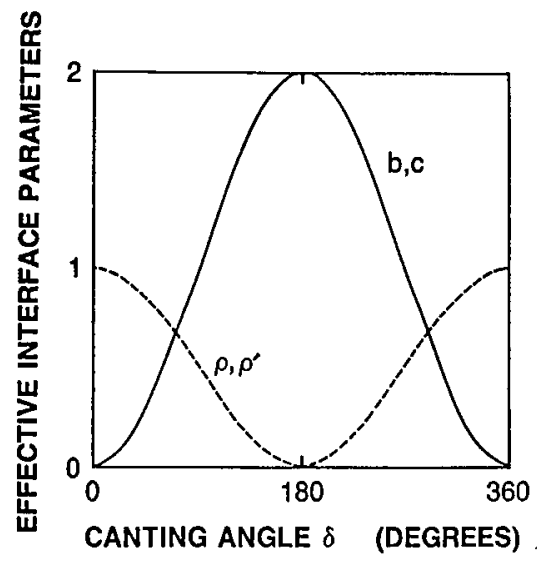

(b)

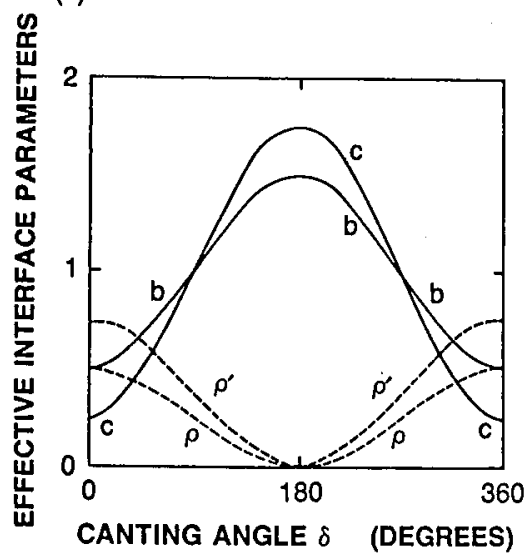

Fig. 3. Numerical examples to illustrate the dependence of the effective interface coupling parameters $\rho$ and $\rho^{\prime}$ (broken curves) and the effective interface pinning parameters $b$ and $c$ (full curves) on the canting angle $\delta$. We have assumed a simple cubic structure for both films, $K_{i}^{A}=K_{i}^{B}=0$, and $S^{A}=S^{B}$. Two cases are considered for the exchange interactions: (a) $J^{A}=J^{B}=J^{A B}$, (b) $J^{A}=1.5 J^{B}=2 J^{A B}$.

(a)

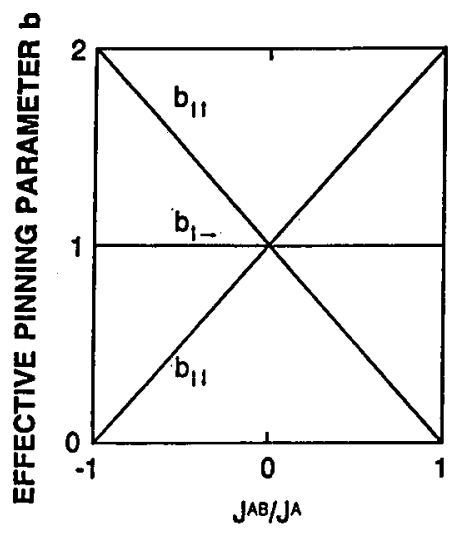

(b)

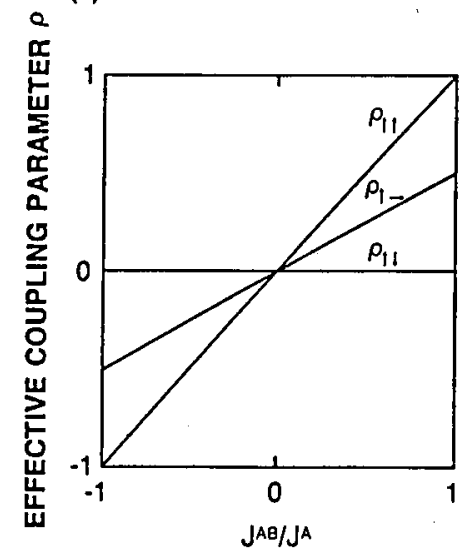

Fig. 4. A numerical example to illustrate the dependence of (a) the effective interface pinning parameter $b$ and (b) the effective interface coupling parameter $\rho$ on $J^{A B} / J^{A}$. Results are shown for three different values of the canting angle corresponding to the magnetization directions being parallel $\left(\delta=0^{\circ}\right)$, perpendicular $\left(\delta=90^{\circ}\right)$, or antiparallel $\left(\delta=180^{\circ}\right)$. We have assumed a simple cubic structure for both films, $\boldsymbol{K}_{i}^{A}=\boldsymbol{K}_{i}^{B}=0$, and $S^{A}=S^{B}$. 
$S^{A}=S^{B}$ and $K_{i}^{A}=0$, as for Fig. 3. Qualitatively similar results are calculated for the dependence of $c$ and $\rho^{\prime}$ on $J^{A B} / J^{B}$. It is seen from Fig. 4(a) that the effective interface pinning parameter $b$ depends linearly on $J^{A B} / J^{A}$, in accordance with (43), and is a constant (independent of $J^{A B} / J^{A}$ ) for the case of perpendicular magnetizations. The effective coupling parameter $\rho$, plotted in Fig. 4(b), is nonzero in general except when $J^{A B}$ is zero, but for the special case of antiparallel magnetizations it is zero for all values of $J^{A B}$, as we discussed beforehand.

In this last case $\left(\delta=180^{\circ}\right)$ our bilayer system is effectively decoupled in two subfilms as regards the spin wave dynamics. It is important to note, however, that in this situation the corresponding pinning parameters $b$ and $c$ are not those for the two subfilms if they existed separately. Instead, $b$ and $c$ are effective parameters that depend on the exchange coupling $J^{A B}$ across the interface. These conclusions may be relevant to some recent experimental studies $[18,19,26,27]$ of bilayer structures consisting of two Fe films that are exchange coupled through a $\mathrm{Cr}$ interlayer, including some cases where the magnetization directions are canted. For example, for an appropriate thickness of $\mathrm{Cr}$, the effective exchange coupling across the interface between the two Fe films is observed to be antiferromagnetic giving $\delta=180^{\circ}$. The experimental observations on this system are consistent with our prediction of an effective dynamic decoupling for the spin waves in the two Fe films. We shall consider further applications of our theory to experiment (particularly for light scattering) in a later work.

The effective interface pinning for general values of the canting angle can easily be understood on re-expressing (43) and (44) as

$$
\begin{gathered}
b=1-\frac{g \mu_{B}}{2 S^{A} J^{A}}\left[K_{i}^{A}+K_{i}^{A: B}\right] \cdot m^{A} \\
c=1-\frac{g \mu_{B}}{2 S^{B} J^{B}}\left[K_{i}^{B}+K_{i}^{B: A}\right] \cdot m^{B},
\end{gathered}
$$

where

$$
K_{i}^{A: B}=\frac{2 J^{A B} S^{B}}{g \mu_{B}} m^{B} \text { and } K_{i}^{B: A}=\frac{2 J^{A B} S^{A}}{g \mu_{B}} m^{A}
$$

are effective mean fields acting on interface spins in film $A(B)$ due to exchange coupling to interface spins in film $B(A)$. Therefore the contributions to $b$ and $c$ can be regarded as arising from the correlation between static components of interface spins. By contrast, we emphasize that our coupling parameters $\rho$ and $\rho^{\prime}$ arise dynamically due to the correlation between tranverse components of the precessing interface spins: they are effective interface coupling parameters for spin waves and are different from the static exchange coupling between the interface spins. Our quoted expressions for $\rho$ and $\rho^{\prime}$ are simplified to some extent because we have made a circular spin-precession approximation (as indicated in Section 3) to obtain the set of finite-difference equations (10). In the absence of this approximation the spin-wave diagonalization procedure for the coupled system would become much more complicated. Hence, there are corrections to $\rho$ and $\rho^{\prime}$ due to ellipticity of the spin precession, but these effects are expected to be small (by analogy with earlier work [28] for single films). 


\section{Acknowledgements}

One of us (H. P.) is indebted to the Center for Interdisciplinary Studies in Chemical Physics at the University of Western Ontario (U.W.O.) for providing the necessary support for his leave in Canada. Thanks are also due to the members of the Physics Department at U.W.O. for their hospitality and to the Institute of Low Temperatures and Structural Studies of the Polish Academy of Sciences for their support.

\section{References}

[1] A. Yelon, in: Physics of Thin Films, vol. 6, eds. M.H. Francombe, R.W. Hoffman, Academic Press, New York 1971, p. 205.

[2] R.E. Camley, T.S. Rahman, D.L. Mills, Phys. Rev. B 27, 261 (1983).

[3] P. Grünberg, K. Mika, Phys. Rev. B 27, 2955 (1983).

[4] L.L. Hinchey, D.L. Mills, Phys. Rev. B 33, 3329 (1986); 34, 1689 (1986).

[5] E.L. Albuquerque, P. Fulco, E.F. Sarmento, D.R. Tilley, Solid State Commun. 58, 41 (1986).

[6] R.E. Camley, M.G. Cottam, Phys. Rev. B 35, 189 (1987).

[7] B. Hillebrands, Phys. Rev. B 37, 9985 (1988).

[8] H. Puszkarski, J.C.S. Lévy, J. Phys., Condens. Malter 2, 4913 (1990).

[9] J.F. Cochran, B. Heinrich, A.S. Arrot, Phys. Rev. B 34, 7788 (1986).

[10] M. Pomerantz, J.C. Slonczewski, E. Spiller, J. Magn. Magn. Matter. 54-57, 781 (1986).

[11] A. Layadi, W.C. Cain, J.-W. Lee, J.O. Artman, IEEE Trans. Magn. MAG-23, 2993 (1987).

[12] C. Vittoria, Phys. Rev. B 37, 2387 (1988).

[13] A. Layadi, J.O. Artman, B.O. Hall, R.A. Hoffman, C.L. Jensen, D.J. Chakrabarti, D.A. Saunders, J. Appl. Phys. 64, 5760 (1988).

[14] M. Grimsditch, M.R. Khan, I.K. Schuller, Phys. Rev. Lett. 51, 498 (1983).

[15] A. Kueny, M.R. Khan, I.K. Schuller, M. Grimsditch, Phys. Rev. B 29, 2879 (1984).

[16] P. Grünberg, Prog. Surf. Sci. 18, 1 (1985).

[17] M.G. Cottam, D.J. Lockwood, Light Scattering in Magnetic Solids, Wiley, New York 1986, p. 190.

[18] C. Carbone, S.A. Alvorado, Phys. Rev. B 36, 2433 (1987).

[19] S.A. Alvorado, C. Carbone, Physica B 149, 43 (1988).

[20] H. Puszkarski, Acta Phys. Pol. A38, 217 (1970).

[21] H. Puszkarski, Acla Phys. Pol. A74, 701 (1988).

[22] H. Puszkarski, Solid State Commun. 72, 887 (1989).

[23] H. Puszkarski, Prog. Surf. Sci. 9, 191 (1979).

[24] R.C. Moul, M.G. Cottam, J. Phys. C 16, 1307 and 1323 (1983). 
[25] G.T. Rado, J.R. Weertman, J. Phys. Chem. Solids 11, 315 (1959).

[26] P. Grünberg, R. Schreiber, Y. Pang, U. Walz, J. Appl. Phys. 61, 3750 (1987).

[27] F. Saurenbach, U. Walz, L. Hinchey, P. Grünberg, W. Zinn, J. Appl. Phys. 63, 3473 (1988).

[28] Diep-The-Hung, H. Puszkarski, J.C.S. Lévy, Vide Couches Minces 204, $313(1980)$. 